\title{
Experimental light scattering by small particles in Amsterdam and Granada
}

\author{
Olga Muñoz ${ }^{1}$, J.W. Hovenier ${ }^{2}$, Fernando Moreno ${ }^{1}$, Daniel Guirado ${ }^{1}$, Hester Volten ${ }^{3}$. \\ ${ }^{1}$ Instituto de Astrofísica de Andalucía, CSIC, Granada, Spain \\ ${ }^{2}$ Astronomical Institute Anton Pannekoek, University of Amsterdam, Amsterdam, The Netherlands. \\ ${ }^{3}$ RIVM- National institute of Public health and the environment, Bilthoven, The Netherlands.
}

\begin{abstract}
We report on two light scattering instruments located in Amsterdam and Granada, respectively. These instruments enable measuring scattering matrices as functions of the scattering angle of collections of randomly orieneted irregular particles. In the past decades, the experimental setup located in Amsterdam, The Netherlands, has produced a significant amount of experimental data. Unfortunately, this setup was officially closed a couple of years ago. We also present a modernized descendant of the Dutch experimental setup recently constructed at the Instituto de Astrofísica de Andalucía (IAA) in Granada, Spain. We give a brief description of the instruments, and present some representative results.
\end{abstract}

\section{Introduction}

Irregular dust particles play an important role in the radiative balance of planetary and cometary atmospheres in the Solar System. Light scattering properties of spherical particles can be easily computed from LorenzMie theory. However, an exact solution for realistic polydispersions of irregular dust particles is extremely difficult if possible at all. Therefore, an experimental study of the scattering behaviour of irregular dust particles that are candidates to be present in different atmospheres is of main importance in order to interpret space- and ground-based observations. For that purpose an experimental setup was built in the 1980s in the group of Hovenier in Amsterdam by Stammes [1] and Kuik [2] and subsequently revised and significantly improved by Volten et al. $[3,4]$.

In the last decades, the experimental setup located in Amsterdam, has produced a significant amount of experimental data that are freely available in a digital form in the Amsterdam Light Scattering Database at http://www.astro.uva.nl/scatter [5,6].Unfortunately, this setup was officially closed a couple of years ago. An improved descendant of the Dutch experimental setup was recently constructed at the Instituto de Astrofísica de Andalucía (IAA) in Granada, Spain.

\section{Experimental setups}

The principle of both setups is similar to that developed by Hunt and Huffman [7].We first discuss de Dutch instrument. A detailed description of this instrument is given by Hovenier [8]. Briefly, a laser is used as a light source. The laser passes through a polarizer and an electro-optic modulator and is subsequently scattered by an ensemble of randomly oriented particles located in a jet stream produced by an aerosol generator. The scattered light is detected by a photomultiplier tube which moves along a ring from about 3 degrees to 174 degrees at two different wavelengths (442 and $633 \mathrm{~nm}$ ). Another photomultiplier tube, the monitor, is located in a fixed position for normalization purposes. Polarization modulation in combination with lock-in detection is used to obtain all elements of the four-by-four scattering matrix as a function of the scattering angle. A photograph of the instrument is presented in Figure 1.

This is an Open Access article distributed under the terms of the Creative Commons Attribution-Noncommercial License 3.0, which permits unrestricted use, distribution, and reproduction in any noncommercial medium, provided the original work is properly cited. 


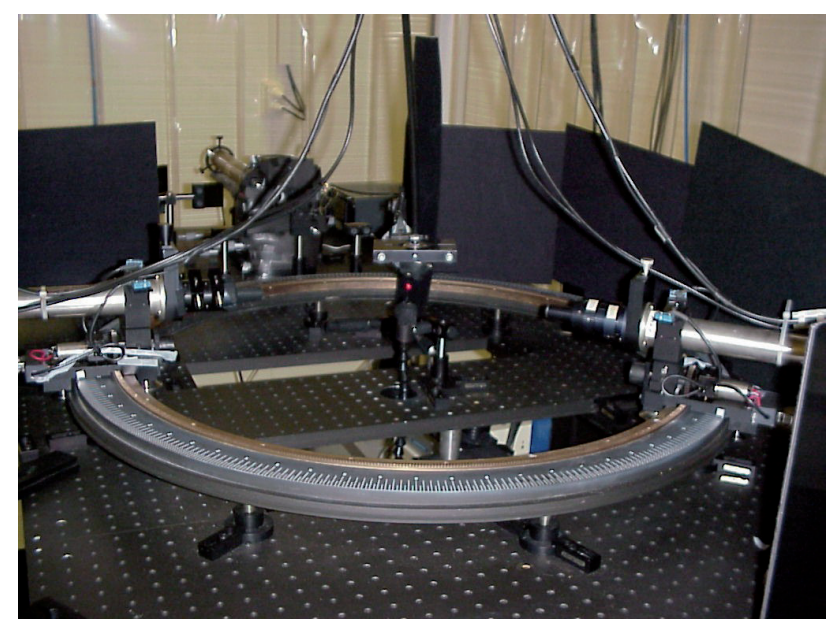

Fig. 1: The Amsterdam light scattering setup.

As mentioned, the Dutch instrument was designed in the 1980's. Therefore, the main improvements of the IAA setup derive from technical developments in the last decades. In the new setup the detectors have been designed so that the blockage of the laser beam at positions close to the forward and backward directions is minimal. This makes it possible to extend the measured scattering angle range from about 3-174 degrees to 3-177 degrees. Moreover, the accuracy of the measurements at small and large scattering angles has been improved by including in the measuring/reduction process corrections for the background signal.

Additionally, we have extended the number of wavelengths of the incident light. In the IAA apparatus, we use a tunable Argon-Krypton laser (483, 488, 520, 568 , and $647 \mathrm{~nm}$ ) as a light source. In Fig. 2 we present a photograph of the Granada light scattering setup.

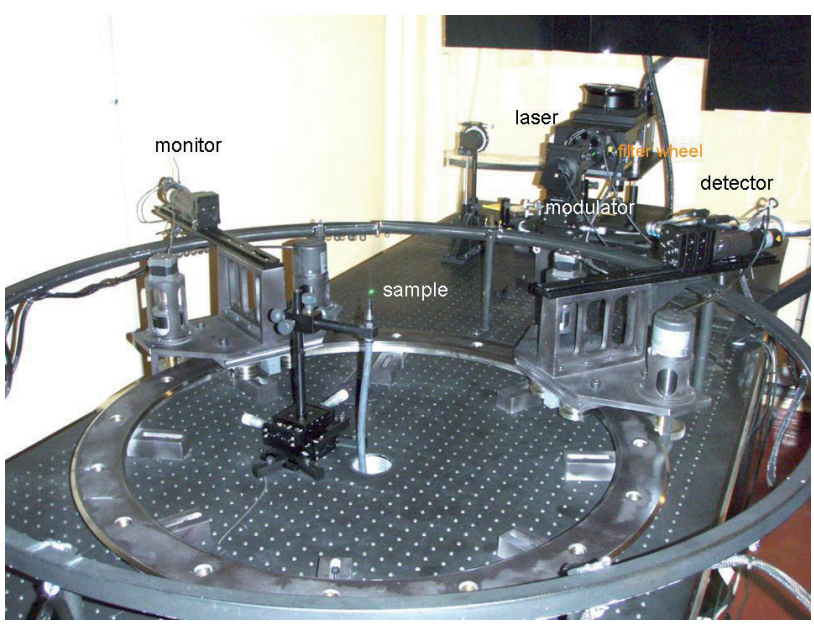

Fig 2: The Granada light scattering setup.

A detailed description of the Granada light scattering setup can be found in [9].

\section{Some examples of measurements}

\subsection{Olivine}

Crystalline Mg-rich olivine (forsterite) is one of the main components of cometary dust [10]. We have experimentally obtained the complete scattering matrices as a function of the scattering angle of three Mg-rich olivine samples [11]. Here we present the measurements for olivine sample $\mathrm{S}$ at $632.8 \mathrm{~nm}$. The size distribution of the olivine sample $\mathrm{S}$ was measured with a Fritsch particle sizer [12], showing diameters mostly in the range 1-5 micrometers. In Fig 3, we present elements of the measured scattering matrix of the olivine sample $\mathrm{S}$ together with Lorenz-Mie calculations for homogeneous optically non-active spherical particles. For the LorenzMie calculations we employed the measured size distribution of the olivine $\mathrm{S}$ sample and its refractive index $\mathrm{m}=1.6+0.00001 \mathrm{i}$ (Dirk Fabian private communication). As shown, dramatic differences between measured and calculated results are found for all scattering matrix elements at nearly all measured scattering angles. Similar differences were found for many other mineral particles, showing that Lorenz-Mie theory can often give very bad results.

Forsterite measurements + Mie calculations

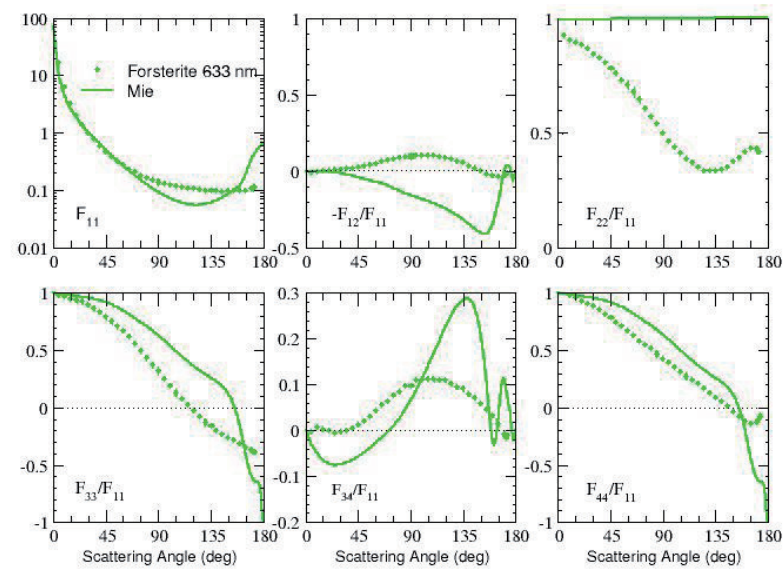

Fig. 3 Measured scattering matrix elements as functions of the scattering angle of olivine $\mathrm{S}$ (forsterite) at 632.8 nm. The measurements are presented together with results of Lorenz-Mie calculations for spheres.

\subsection{Hematite}

Hematite is believed to be an important component of Martian dust [13] and it is also a constituent of terrestrial aerosols [14]. Hematite is a birefrigent material with a large real and imaginary parts of the refractive index at $632.8 \mathrm{~nm}(\mathrm{~m}=3-0.1 \mathrm{i})$. In Fig. 4 we show the measured scattering matrix elements as functions of the scattering angle for the hematite sample at $632.8 \mathrm{~nm} \mathrm{[15].} \mathrm{The}$ measurements are presented together with Lorenz-Mie 
calculations for the same size distribution and refractive index as our hematite sample has. Here the differences between measured and calculated results are much smaller than in Fig.
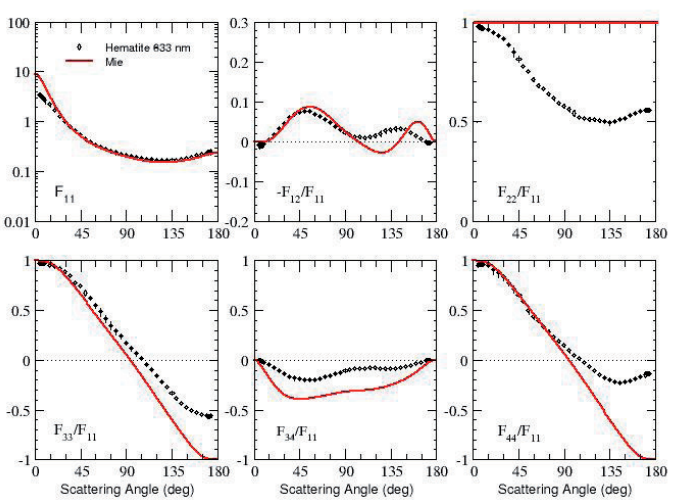

Fig. 4 Measured scattering matrix elements as functions of the scattering angle of hematite at $632.8 \mathrm{~nm}$. The measurements are presented together with results of Lorenz-Mie calculations for spheres.

As discussed in [15] both the experimental and theoretical results seem to indicate that the scattering behaviour of irregular mineral particles that have a high real part of the refractive index is not very dependent on the shape of the particles. In this case, Lorenz-Mie theory may give reasonable results despite the irregular shapes of the particles.

\section{References}

1 Stammes P. Light scattering properties of aerosols and the radiation inside a planetary atmosphere. Ph.D. Dissertation 1989, Free Univesity, Amsterdam.

2 Kuik F. Single scattering by ensembles of particles with various shapes. Ph. D. Dissertation 1992, Free University, Amsterdam.

3 Volten H. Light scattering by small particles. An experimental study. Ph. D. Dissertation 2001, Free University, Amsterdam.

4 Volten H, Muñoz O, Rol E, de Haan JF, Vassen V, Hovenier JW, Muinonen K, Nousianen T. J Geophys Res 2001;106:17375-17401.

5 Volten H, Muñoz O, Hovenier JW, de Haan JF, Vassen W, van der Zande WJ, Waters LBFM. JQSRT 2005;90(2):191-206.
6 Volten H, Muñoz O, Hovenier JW, Waters LBFM. JQSRT 2006;100:437-443.

7 Hunt AJ, Huffman DR. Rev. Sci. Instrum., 44, No $12,1753-1762,(1973)$.

8 Hovenier JW. Measuring scattering matrices of small particles at optical wavelengths. In: Light scattering by nonspherical particles, edited by M. I. Mishchenko, J. W. Hovenier, and L. D. Travis, Academic, San Diego, CA, 2000:355-365.

9 Muñoz O., Moreno, F, Guirado D., Ramos. J.L., López, A., Girela, F., Jerónimo, J.M., Costillo, L.P., and Bustamante, I. JQSRT;2010: 11: 187-196.

10 Hanner , M.S.,Lynch, D.K., and Rusell, R.W.Astrophys;1994:274-285.

11 Muñoz O., Volten H., de Hann J.F., Vassen W., and Hovenier J. A\&A;2000:360:777-788.

12. Konert M., and Vandenberghe J. Sedimentology. 1997;44:523-535.

13. Morris R.V., and Lauer, J.Geophys. Res. 1990; 94: 5101-5109.

14. Sokolik I.N., and Toon O.B., J. Geophys. Res. 1999; 104:9243-9444.

15. Muñoz O., Volten H., Hovenier J.W., Min M., Shkuratov Y.G., Jalava J.P. van der Zande W.J., and Waters L.B.F.M. A\&A. 2006; 446: 525-535. 